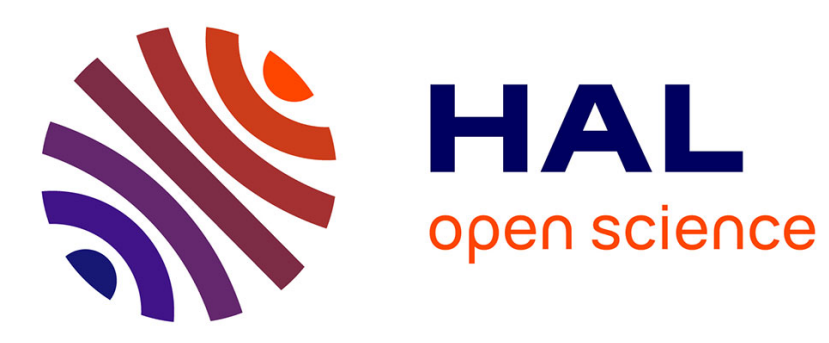

\title{
Les pratiques sportives des adolescents: une différenciation selon le genre
}

Paul Fontayne, Philippe Sarrazin, Jean-Pierre Famose

\section{To cite this version:}

Paul Fontayne, Philippe Sarrazin, Jean-Pierre Famose. Les pratiques sportives des adolescents: une différenciation selon le genre. STAPS: Revue internationale des sciences du sport et de l'éducation physique, 2001, 55, pp.23-37. 10.3917/sta.055.0023 . hal-00387231

\section{HAL Id: hal-00387231 \\ https://hal.science/hal-00387231}

Submitted on 25 May 2009

HAL is a multi-disciplinary open access archive for the deposit and dissemination of scientific research documents, whether they are published or not. The documents may come from teaching and research institutions in France or abroad, or from public or private research centers.
L'archive ouverte pluridisciplinaire HAL, est destinée au dépôt et à la diffusion de documents scientifiques de niveau recherche, publiés ou non, émanant des établissements d'enseignement et de recherche français ou étrangers, des laboratoires publics ou privés. 


\title{
Les pratiques sportives des adolescents : une différenciation selon le genre
}

\author{
Paul Fontayne ${ }^{1}$, Philippe Sarrazin² ${ }^{2}$ Jean-Pierre Famose ${ }^{1}$ \\ ${ }^{1}$ Université de Paris XI-Orsay, Division STAPS, \\ Centre de Recherches en Sciences du Sport \\ ${ }^{2}$ Université Joseph Fourier, UFR-APS, \\ Laboratoire «Etudes et Recherches sur l'Offre Sportive »
}

\begin{abstract}
$\underline{\text { Résumé }}$
L'identité sexuelle des sujets influence la valeur que ceux-ci accordent aux activités (Eccles, Adler, Futterman, Goff, Kaczala, Meece, \& Midgley, 1983). Dans cette étude, nous essayons de montrer que le choix des pratiques sportives de 858 sujets adolescents (403 garçons et 455 filles), âgés en moyenne de 15.4 ans $(E T=0.83)$ est lié à leur profil de genre (Bem, 1981). Des analyses statistiques (Chi2, MANOVAs, Analyse en Composantes Multiples) furent réalisées à partir d'un questionnaire dans lequel les sujets devaient (a) répondre à une version courte du BSRI (Fontayne, Sarrazin, \& Famose, 2000), (b) indiquer leur sexe, leur pratique sportive, et la modalité de cette pratique (compétitive / non-compétitive). Les résultats montrent que la perception d'un sport comme étant conforme aux rôles sexués masculin ou féminin influence le choix des pratiques. Les sujets « masculins » et «féminins » choisissent des sports congruents et rejettent les sports non congruents avec leur identité, tandis que les sujets « androgynes » et «non-différenciés » révèlent plus aisément des choix non conformes aux stéréotypes culturels de leur sexe.
\end{abstract}

Mots-clés : sport, genre, valeur.

\section{Sport practices of French teenagers : a gender differenciation}

\begin{abstract}
$\underline{\text { Abstract }}$
The sexual identity of the subjects influences the value they concede to the activities (Eccles, Adler, Futterman, Goff, Kaczala, Meece, \& Midgley, 1983). The purpose of this study is to examine whether the choice about sports activities of 858 teen-agers ( 403 boys and 455 girls) is linked to their gender-role (Bem, 1981). Statistical analysis (Chi2, MANOVAs, Multiple Components Analysis) were conducted from the answers of the subjects to : (a) the French short version of the BSRI (Fontayne, Sarrazin, \& Famose, 2000), (b) a questionnaire where the subjects have to indicated their sex, their sport activity, and the type of this practice (competition / non-competition). The results show that the gender-based schematic processing affects the choice of the sport practice. The « masculine » and « feminine » subjects prefer sex-appropriate and avoid sex-inappropriate sports. The « androgynous » and « undifferenciated » subjects are more engaged in cross-sexual or «neutral » sports than the sex-typed subjects.
\end{abstract}

Key words : sport, gender, value. 


\section{INTRODUCTION}

Depuis une vingtaine d'années, une littérature abondante s'est développée autour de la conceptualisation des rôles sociaux sexuellement typés. L'hypothèse théorique fondamentale est la suivante : chaque culture oriente et encourage certaines conduites, traits et activités considérés comme des caractéristiques propres à chacun des sexes (Cross \& Madson, 1997). C'est la raison pour laquelle, les concepts de masculinité et de féminité sont utilisés pour faire référence aux rôles ou aux traits psychologiques attachés respectivement aux hommes et aux femmes. Si, dans les premières formulations théoriques, on présumait que les concepts de masculinité et de féminité se situaient aux deux extrémités d'un continuum unique, il existe maintenant un large consensus autour de l'hypothèse de Constantinople (1973) selon laquelle, masculinité et féminité constituent deux dimensions indépendantes. Chaque individu peut posséder un niveau plus ou moins élevé de ces deux traits, quel que soit son sexe biologique (Alain, 1996 ; Blanchard-Fields, Suhrer-Roussel, \& Hertzog, 1994). A l'instar de Bem (1981), on parle d'individus typés « masculin » (qui endossent les rôles et traits psychologiques caractéristiques des hommes et rejettent ceux qui caractérisent les femmes), d'individus typés «féminin » (qui endossent les rôles et traits psychologiques caractéristiques des femmes et rejettent ceux qui caractérisent les hommes), d'individus « androgynes » (qui endossent les rôles et traits psychologiques qui caractérisent à la fois les hommes et les femmes), et d'individus «non-différenciés » (qui ne marquent pas d'appétence particulière pour les rôles et traits psychologiques caractéristiques des hommes ou des femmes).

L'importance de ces rôles sexués a été soulignée dans plusieurs modèles théoriques (Bem, 1981, 1985 ; Cross \& Madson, 1997 ; Wigfield \& Eccles, 1992). Une fois intériorisés, ils constituent de véritables « schémas de soi » liés au genre qui servent de filtres cognitifs pour interpréter les événements et orienter les conduites (Bem, 1981, 1985). Par exemple, dans leur modèle «Expectation - Valence », Eccles et ses col- laborateurs (Eccles, Adler, Futterman, Goff, Kaczala, Meece, \& Midgley, 1983), ont montré que le choix d'une activité d'accomplissement (une discipline scolaire, une activité sportive, etc.) dépendait directement des attentes de succès des individus, ainsi que de la valeur qu'ils accordent à la tâche (ou activité). Selon eux, les attentes et les valeurs seraient elles-mêmes déterminées en partie par l'identité sexuelle. Les garçons ont tendance à valoriser plus que les filles les activités qu'ils estiment conformes à leur sexe. En outre, ils les trouvent moins difficiles et s'attendent davantage à réussir dans ces activités. C'est ainsi que le curriculum scolaire des sujets est influencé par le «typage sexuel » des activités (pour revue, voir Duru-Bellat, 1994, 1995).

Le sport en tant que pratique sociale ne fait pas exception à ce marquage sexuel. En général, il est considéré par les auteurs comme un domaine plutôt masculin (Davisse \& Louveau, 1991, 1998 ; Harry, 1995 ; Matteo, 1986, 1988 ; Messner, 1988, 1990 ; Ryckman \& Hamel, 1995). Certains auteurs ont vu là une des explications aux différences de participation et d'investissement entre les garçons et les filles (Eccles \& Harold, 1991; Deeter, 1989, 1990). Pour ces chercheurs, ces différences qui paraissent émerger très tôt (Eccles, Jacobs, \& Harold, 1990 ; Tap, 1985 ; Wigfield, Harold, Freedman-Doan, Eccles, Yoon, Arbreton, \& Blumenfeld, 1997) semblent être plutôt la conséquence d'une socialisation des rôles sexués que d'une différence d'aptitudes naturelles. L'environnement social et culturel inculquerait très tôt ce qu'il est approprié de faire en fonction du sexe. Cette socialisation aboutirait à ce que les sujets s'engagent dans des activités singulières en fonction de leur conformité aux stéréotypes de leur genre. Mais le sport ne recouvre pas une modalité de pratique unique. Il est pluriel : pratique compétitive ou de loisir, collective ou individuelle, d'affrontement ou « esthétique », cadre fédéral ou modalité libre, etc. (Pociello, 1995). Même si les choses ont considérablement évolué depuis ces trente dernières années - les femmes pratiquant de plus en plus d'activités sportives - le type de sport et les modalités de la pratique demeurent différentes entre les 
hommes et les femmes (Davisse \& Louveau, 1998). Sur la base de l'appropriation sexuée des pratiques, plusieurs travaux ont corroboré l'existence d'activités sportives masculines, féminines ou appropriées au deux sexes (Czisma, Wittig, \& Schurr, 1988 ; Ignico, 1989 ; Koivula, 1995 ; Matteo, 1986, 1988 ; Salminen, 1990). Si l'appropriation différenciée des pratiques en fonction du sexe des individus a suscité un grand nombre de travaux, il n'existe à notre connaissance aucune étude qui appréhende les effets du genre (ou de la sexuation psychologique) des individus sur cette appropriation. Conformément aux travaux de Bem (1981), nous pensons pouvoir avancer que les individus possédant une forte identité de genre (i.e., Typés Masculins ou Typés Féminins) devraient marquer une préférence pour les activités sportives congruentes avec cette identité, et ce, quel que soit leur sexe biologique.

En fonction de ce qui vient d'être énoncé, nous formulerons les hypothèse suivantes :

- Hypothèse 1. Les garçons devraient plus nombreux que les filles à pratiquer une activité sportive.

- Hypothèse 2. La pratique sportive se distribue différemment selon le genre. Concrètement, nous nous attendons à ce que les sujets Masculins et Androgynes soient plus sportifs que les sujets Féminins et Non-différenciés.

- Hypothèse 3. En cas de pratique sportive, les sujets devraient se répartir suivant les mêmes patrons que dans les hypothèses 1 et 2 en fonction de la modalité de pratique. Pratiquement, nous pensons que les garçons devraient se révéler plus compétitifs que les filles, tandis que, dans le même temps, les sujets Masculins et Androgynes devraient également montrer plus d'appétence pour les pratiques compétitives que les sujets Féminins et Non-différenciés.

- Hypothèse 4. La perception d'une activité sportive comme étant conforme aux rôles masculins et féminins influence le choix des pratiques en fonction du genre $\mathrm{du}$ sujet. Les sujets Masculins devraient plus volontiers s'engager dans des pratiques masculines et éviter de s'engager dans des activités perçues comme féminines. L'inverse devrait être mis en évidence pour les sujets Féminins (i.e., éviter la pratique sportive et les sports masculins, ou bien s'engager dans des pratiques sportives féminines). En ce qui concerne les individus dont le schème de catégorisation serait absent (i.e., Non-différenciés), ou ceux qui n'utiliseraient pas cette dichotomie du masculin et du féminin (i.e., Androgynes), on s'attend à ce qu'ils pratiquent plus aisément des activités nonconformes aux stéréotypes de leur sexe, ou qu'ils soient indifférents à ces pratiques.

- Hypothèse 5 : Enfin, nous présumons que, pour le groupe de sujets étudié, le champ des pratiques sportives se projette sur un espace défini à partir des deux axes orthogonaux déterminant leur profil de genre.

\section{MÉTHODE}

\subsection{Sujets}

La population était constituée de 403 garçons et de 455 filles issus de classe de troisième de sept collèges de la banlieue ouest de Paris et d'un collège de la Drôme. Les sujets étaient âgés en moyenne de 15.4 ans $(\mathrm{ET}=0.83)$, et étaient tous volontaires pour remplir les questionnaires dont la présentation avait auparavant été soumise à autorisation parentale.

\subsection{Procédure}

Les questionnaires étaient administrés dans une classe, à la fin d'un cours d'éducation physique sous la responsabilité conjointe du professeur d'E.P.S. de la classe et d'un chercheur en psychologie du sport. Ce dernier annonçait qu'il menait une enquête sur la pratique des activités physiques et sportives, et notamment sur le rapport qu'il pouvait exister entre les traits de caractères de chacun et le type de sports pratiqués. Il était expressément mentionné aux sujets que l'anonymat était garanti. La durée pour répondre aux questionnaires était d'environ 20 minutes.

\subsection{Mesures}

2.3.1. Le genre. Le genre du sujet était déterminé par le score aux échelles de masculinité et de féminité de la version courte pour adolescents français du Bem Sex-Role 
Inventory (Fontayne, Sarrazin, \& Famose, 2000). Le questionnaire est constitué de 18 items, représentant chacun un attribut de personnalité. Certains sont caractéristiques de traits féminins [e.g., Je suis toujours prêt(e) à écouter les autres; je suis affectueux (se)] et d'autres de traits masculins [e.g., Je me comporte en chef; Je suis sûr(e) de moi]. Le sujet est invité à indiquer pour chacun d'eux, s'il est particulièrement approprié pour le décrire. Il utilise pour cela une échelle de type Likert en 7 points : (1) « Jamais vrai » et (7) « Toujours vrai ». Dans les travaux antérieurs menés avec plus de 1200 adolescents (Fontayne et al., 2000), cette version raccourcie du BSRI a témoigné d'une bonne validité de construit, et les deux sous-échelles ont démontré une consistance interne élevée et une fiabilité testretest acceptable. Des preuves relatives à la validité concomitante, et à la validité prédictive du questionnaire ont aussi été rapportées. Pour cet échantillon de sujets, les coefficients alpha (Cronbach, 1951) sont de .81 et de .86 respectivement pour les sous-échelles masculine et féminine.

Une fois effectuée la moyenne des items de chaque sous-échelle, la méthode du partage par la médiane (median split) fut utilisée pour déterminer le genre des sujets (Bem, 1981). Les sujets furent considérés comme (a) Typés Masculins, quand ils obtenaient des scores supérieurs à la médiane à l'échelle de Masculinité et inférieurs à la médiane à l'échelle de Féminité, (b) Typés Féminins, quand ils obtenaient des scores supérieurs à la médiane à l'échelle de Féminité et inférieurs à la médiane à l'échelle de Masculinité, (c) Androgynes, quand ils obtenaient des scores supérieurs à la médiane aux deux sous-échelles, et (d) Non-différenciés, quand ils obtenaient des scores inférieurs à la médiane aux deux sous-échelles.

2.3.2. La pratique sportive. Il a été décidé de retenir l'acception associative et compétitive de la pratique sportive. Pour cela, il était demandé aux sujets d'indiquer s'ils pratiquaient une activité physique dans le cadre d'une association sportive ou d'un club. La question était libellée de la façon suivante : «Pratiques- tu un sport en club ou en association?

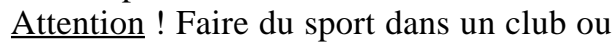
dans une association signifie que tu es inscrit dans une structure, et que tu as payé une cotisation pour bénéficier de ses services (installations, cours, compétitions, etc.). Les sujets qui déclaraient pratiquer un sport dans un club furent classés comme «Sportifs » (SP), les autres comme « Non-Sportifs » (NSP). Les sujets devaient indiquer s'ils pratiquaient cette activité sportive en compétition et, le cas échéant, le niveau de pratique (i.e., interclubs, départemental, régional, etc.). Les sujets sportifs (SP) étaient alors répartis en deux nouvelles catégories : « Sportifs Compétitifs » (SPC) et «Sportifs Non-Compétitifs » (SPNC).

Quand un sujet pratiquait une activité sportive, le «typage sexuel » (Hurtig \& Pichevin, 1986) de celle-ci était effectué. Pour cela, les études antérieures effectuées pour évaluer le degré de «masculinité » ou de «féminité » d'une pratique sportive, ont été utilisées (Colley, Nash, O'Donnel, \& Restorick, 1987; Hall, Durborow, \& Progen, 1986 ; Ignico, 1989 ; Kirckaldy \& Furnham, 1990 ; Koivula, 1995 ; Matteo, 1986, 1988 ; Ostrow, Jones, \& Spiker, 1981; Salminen, 1990). En général, dans ces études la procédure consiste à établir une liste relativement exhaustive de sports et de demander aux sujets s'ils considèrent chacun comme approprié aux hommes (e.g., boxe, football, rugby, etc.), approprié aux femmes (e.g., danse, gymnastique, équitation, etc.), ou comme pouvant être pratiqué par les deux sexes (e.g., tennis, natation, course à pied, etc.). Certains auteurs effectuent cette typologie de manière indirecte, à partir du nombre de licenciés fédéraux (Davisse \& Louveau, 1998). Bien que quelques changements dans le classement de certaines pratiques puissent être constatés notamment en fonction de l'âge (Marin, 1988), les travaux font état d'une relative homogénéité des classifications, quels que soient les pays occidentaux (Archer \& McDonald, 1990 ; Carvalho, Smith, Hunter, \& Costabile, 1990 ; Kirckaldy \& Furnham, 1990). Le tableau I présente la répartition des activités sportives des sujets en fonction de leur typage sexuel. 
TABLEAU 1 : Répartition des garçons et des filles en fonction du type pratique sportive (en fréquence).

\begin{tabular}{|c|c|c|c|c|c|c|c|c|c|c|c|}
\hline \multicolumn{4}{|c|}{ Activités masculines } & \multicolumn{4}{|c|}{$\begin{array}{c}\text { Activités appropriées } \\
\text { aux deux sexes }\end{array}$} & \multicolumn{4}{|c|}{ Activités féminines } \\
\hline Sports & G & $\mathrm{F}$ & Total & Sports & $\mathrm{G}$ & $\mathrm{F}$ & Total & Sports & $\mathrm{G}$ & $\mathrm{F}$ & Total \\
\hline Aviron & 1 & 2 & 3 & Aïkido & 4 & 1 & 5 & Aérobic & 0 & 1 & 1 \\
\hline Base-Ball & 1 & 2 & 3 & Athlétisme & 4 & 7 & 11 & Danse & 0 & 82 & 82 \\
\hline Basket Ball & 21 & 6 & 27 & Badminton & 15 & 18 & 33 & Danse Folklo. & 0 & 1 & 1 \\
\hline Boxe & 0 & 1 & 1 & Course Orient. & 2 & 2 & 4 & Équitation & 7 & 39 & 46 \\
\hline Boxe Franç. & 5 & 0 & 5 & Escrime & 3 & 1 & 4 & GRS & 0 & 9 & 9 \\
\hline Boxe Thaï & 4 & 0 & 4 & Golf & 4 & 1 & 5 & Gymnastique & 3 & 10 & 13 \\
\hline Canoé-Kayak & 1 & 0 & 1 & Jiu-Jitsu & 2 & 1 & 3 & Natation Syn. & 0 & 2 & 2 \\
\hline Cyclisme & 5 & 0 & 5 & Natation & 12 & 17 & 29 & Streching & 0 & 1 & 1 \\
\hline Escalade & 5 & 3 & 8 & Tennis & 32 & 34 & 66 & Twirling Bât. & 0 & 1 & 1 \\
\hline Football & 52 & 1 & 53 & Volley-Ball & 17 & 18 & 35 & & & & \\
\hline Handball & 7 & 3 & 10 & & & & & & & & \\
\hline Hockey/Gazon & 2 & 1 & 3 & & & & & & & & \\
\hline Hockey/Glace & 1 & 0 & 1 & & & & & & & & \\
\hline Judo & 9 & 3 & 12 & & & & & & & & \\
\hline Karaté & 7 & 0 & 7 & & & & & & & & \\
\hline Kempo & 1 & 0 & 1 & & & & & & & & \\
\hline Kick Boxing & 1 & 0 & 1 & & & & & & & & \\
\hline Kung Fu & 3 & 1 & 4 & & & & & & & & \\
\hline Moto & 0 & 1 & 1 & & & & & & & & \\
\hline Musculation & 4 & 0 & 4 & & & & & & & & \\
\hline Parachutisme & 1 & 0 & 1 & & & & & & & & \\
\hline Patinage Vite. & 1 & 0 & 1 & & & & & & & & \\
\hline Plongée & 3 & 2 & 5 & & & & & & & & \\
\hline Roller & 1 & 0 & 1 & & & & & & & & \\
\hline Rugby & 15 & 0 & 15 & & & & & & & & \\
\hline Tae Kwon Do & 5 & 1 & 6 & & & & & & & & \\
\hline Tennis Table & 9 & 2 & 11 & & & & & & & & \\
\hline Tir & 1 & 1 & 2 & & & & & & & & \\
\hline Tir Arc & 3 & 2 & 5 & & & & & & & & \\
\hline Viet Vo Dao & 1 & 0 & 1 & & & & & & & & \\
\hline Vo Dan Toc & 2 & 0 & 2 & & & & & & & & \\
\hline VTT & 5 & 1 & 6 & & & & & & & & \\
\hline Water Polo & 2 & 0 & 2 & & & & & & & & \\
\hline Total & 179 & 33 & 212 & & 95 & 100 & 195 & & 10 & 146 & 156 \\
\hline
\end{tabular}

Pour la plupart des sports, la classification dans l'une des trois catégories s'est faite sans difficulté. Cependant quelques activités ont été plus difficiles à classer, soit parce qu'elles n'ont jamais été citées dans les travaux antérieurs (c'est le cas de l'aïkido et d'autre sports de combat rassemblant pour la plupart des techniques de combat pieds-poings); soit parce que la dénomination de ces activités est en contradiction avec le rapport Hommes - Femmes dans le nombre de licenciés au sein de la fédération organisant ce sport en France (c'est le cas du tennis). Les choix ont été faits à partir des critères suivants : (a) conformité aux travaux antérieurs (par exemple, le tennis fut classé comme une activité appropriée aux deux sexes), (b) conformité aux études sur les pratiques dans le champ culturel sportif français (Arnaud \& Terret, 1996 ; Clément, 1981 ; Davisse \& Louveau, 1991, 1998 ; Pociello, 1981, 1995; Vigarello, 1988) (par exemple, les pratiques de combat les plus euphémisées comme l'aïkido et l'escrime ont été classées comme étant appropriées aux deux sexes, alors que toutes les autres furent classées comme étant masculines, et 
TABLEAU II : Critères d'appropriation ou de non-appropriation des pratiques sportives selon le sexe.

\begin{tabular}{|c|c|c|}
\hline & Activités masculines ou non-féminines & Activités féminines \\
\hline Ignico (1989) & $\begin{array}{l}\text { - vitesse } \\
\text { - force } \\
\text { - stratégie } \\
\text { - propulsion d'objets } \\
\text { - endurance }\end{array}$ & $\begin{array}{l}\text { - jeux à tour de rôles } \\
\text { - expressivité } \\
\text { - peu de règles } \\
\text { - peu de joueurs } \\
\text { - activité motrice fine ou partielle }\end{array}$ \\
\hline Kane (1988) & $\begin{array}{l}\text { - essaye de battre l'adversaire } \\
\text { par la force physique } \\
\text { - utilise des objets lourds } \\
\text { - engagement dans une compétition } \\
\text { face à face, dans laquelle les corps } \\
\text { peuvent entrer en contact }\end{array}$ & $\begin{array}{l}\text { - corps projeté dans l'espace } \\
\text { avec un but esthétique } \\
\text { - objets légers } \\
\text { - barrière spatiale pour prévenir } \\
\text { le contact physique }\end{array}$ \\
\hline Louveau $(1991,1998)$ & $\begin{array}{l}\text { - montrer ou exercer sa force } \\
\text { - se livrer à un combat dur et } \\
\text { rapproché } \\
\text { - porter ou recevoir des coups } \\
\text { - jouer à la balle au pied sur un grand } \\
\text { terrain } \\
\text { - manipuler des armes } \\
\text { - s'engager dans des efforts intenses } \\
\text { de longues durées } \\
\text { - piloter des engins mécaniques } \\
\text { ou motorisés } \\
\text { - prendre des risques dans des } \\
\text { pratiques agoraphiles }\end{array}$ & \\
\hline Metheny (1965) & $\begin{array}{l}\text { - objets lourds } \\
\text { - contacts corporels } \\
\text { - opposition face à face } \\
\text { - projection du corps de l'athlète } \\
\text { à travers l'espace pour un temps } \\
\text { relativement long }\end{array}$ & $\begin{array}{l}\text { - objets légers } \\
\text { - précision mais pas force } \\
\text { - peu de contacts corporels } \\
\text { - patrons esthétiques du vol corporel }\end{array}$ \\
\hline
\end{tabular}

(c) conformité aux critères définissant une pratique sportive masculine et/ou féminine (voir tableau II).

\section{RÉSULTATS}

\subsection{Pratique versus non-pratique sportive}

Un premier test du Chi-carré fut effectué avec comme variable dépendante (VD) le sexe du sujet et comme variable indépendante (VI) la pratique sportive (SP) ou la non-pratique sportive (NSP). Les résultats indiquent que la répartition des sujets s'écarte trop de la distribution théorique pour être le résultat du hasard : $\chi^{2}(1, \mathrm{~N}=858)=$ $8.38, p<.01$. L'analyse de la contribution des cases a posterori montre que les adolescents sont significativement plus nombreux que les adolescentes à pratiquer un sport.
Un autre test du Chi-carré fut réalisé avec comme VD le genre du sujet et comme VI la pratique sportive. La répartition des sujets SP et NSP en fonction de leur profil lié au genre s'écarte de manière très significative la distribution théorique : $\chi^{2}(3, \mathrm{~N}=$ $858)=39.20, p<.0001$. L'analyse de la contribution des cases a posteriori révèle que les sujets Masculins et Androgynes sont sur-représentés, tandis que les sujets Féminins et Non-différenciés sont sousreprésentés parmi les SP. Une répartition inverse est constatée parmi les NSP (voir tableau III). Les mêmes résultats sont observés pour chacun des deux sexes : $\chi^{2}$ Garçons $(3, \mathrm{~N}=403)=20.77, p<.0001 ; \chi^{2}$ Filles $(3$, $\mathrm{N}=455)=15.24, p<.002$. Pour chacune des deux populations, l'étude de la contribution des cases a posteriori révèle une répartition similaire des sujets en fonction de leur genre que pour la population totale. 
TABleau 3 : Répartition, selon le genre, des sujets « Sportifs » et « Non Sportifs ».

\begin{tabular}{|ccccc|}
\hline & Masculin & Androgyne & Féminin & Non-différencié \\
\cline { 2 - 5 } Sportifs & $158(74.88 \%)$ & $158(77.07 \%)$ & $114(53.27 \%)$ & $134(58.77 \%)$ \\
Non sportifs & $53(25.12 \%)$ & $47(22.93 \%)$ & $100(46.73 \%)$ & $94(41.23 \%)$ \\
\hline Note $:$ Les chiffres entre parenthèses correspondent aux pourcentages par colonne. \\
\hline
\end{tabular}

Une MANOVA* fut conduite avec : (a) la pratique sportive (SP vs NSP), (b) le sexe, (c) le genre comme VI, et le score aux deux sous-échelles de Féminité et de Masculinité de la version courte du BSRI comme VD.

L'analyse multivariée révèle : (a) un effet principal de la variable pratique sportive significatif, Lambda de Wilks (2, 841) $=.98, p<.001$, (b) un effet principal de la variable sexe significatif, Lambda de Wilks $(2,841)=.94, p<.0001$, (c) un effet principal de la variable genre significatif, Lambda de Wilks $(6,1682)=.22, p<$ .0001 , (d) une absence d'interaction significative entre la variable pratique sportive et la variable sexe, Lambda de Wilks (2, $841)=.996, p=.56$, (e) une absence d'interaction significative entre la variable pratique sportive et la variable genre, Lambda de Wilks $(2,1682)=.99, p=.08$, (f) une interaction significative entre la variable genre et la variable sexe, Lambda de Wilks $(6,1682)=.98, p<.01,(\mathrm{~g})$ une absence d'interaction significative entre les variables pratique sportive, sexe et genre, Lambda de Wilks $(6,1682)=.995, p=.67$.

Les tests univariés font apparaître : (a) un effet significatif au niveau de l'échelle de Masculinité : $F(1,854)=59.54, p<$ .0001 , les SP $(M=4.81)$ obtenant un score plus élevé sur cette échelle que les NSP ( $M$ $=4.17$ ), et (b) une absence d'effet significatif au niveau de l'échelle de Féminité : $F$ $(1,854)=.24, p=.62$.

\subsection{Compétition versus non-compétition}

Un test du Chi-carré fut réalisé avec comme VD le genre du sujet et comme VI la pratique compétitive (SPC) ou non-compétitive (SPNC). La répartition des sujets de cette étude s'écarte de manière très significative de la distribution théorique : $\chi^{2}(3, \mathrm{~N}=564)=23.79, p<.0001$. Une étude de la contribution des cases a posteriori montre que si les sujets Masculins et Féminins sont respectivement sur-représentés et sous-représentés chez les SPC (la répartition inverse étant également constatée chez les sujets SPNC), la répartition des sujets Androgynes et Non-différenciés n'est pas significativement différente de la distribution attendue, ni chez les SPC, ni chez les SPNC (voir tableau IV).

Une MANOVA fut conduite avec : (a) la modalité de pratique sportive (SPC vs SPNC), (b) le genre, (c) le sexe comme VI, et le score aux deux sous-échelles de Féminité et de Masculinité de la version courte du BSRI comme VD.

TABLEAU 4 : Répartition, selon le genre, des sujets « Sportifs Compétitifs » et « Sportifs Non-Compétitifs ».

\begin{tabular}{|ccccc|}
\hline & Masculin & Androgyne & Féminin & Non-différencié \\
\cline { 2 - 5 } & $125(79.11 \%)$ & $110(69.62 \%)$ & $59(51.75 \%)$ & $85(63.43 \%)$ \\
Sportifs Compétitifs (SPC) & $33(20.89 \%)$ & $48(30.38 \%)$ & $55(48.25 \%)$ & $49(36.57 \%)$ \\
Sportifs Non-Compétitifs (SPNC) & & & \\
\hline Note : Les chiffres entre parenthèses correspondent aux pourcentages par colonne. & & \\
\hline
\end{tabular}

* Nous faisons apparaître les résultats d'un Chi2 et d'une MANOVA parce que chacune de ces procédures renvoie à des modèles théoriques différents de l'androgynie psychologique (pour revue, voir Alain, 1996). La pertinence de chacune de ces approches étant encore actuellement discutée, nous analyserons donc nos données à la lumière de ces deux méthodes statistiques. 
L'analyse multivariée révèle : (a) un effet principal de la variable modalité de pratique significatif, Lambda de Wilks (2, 547 ) $=.95, p<.0001$, (b) un effet principal de la variable sexe significatif, Lambda de Wilks $(2,547)=.94, p<.0001$, (c) un effet principal de la variable genre significatif, Lambda de Wilks $(6,1094)=.23, p<$ .0001 , (d) une absence d'interaction significative entre la variable modalité de pratique et la variable sexe, Lambda de Wilks $(2,547)=.997, p=.47$, (e) une absence d'interaction significative entre la variable pratique sportive et la variable genre, Lambda de Wilks $(6,1094)=.99, p=.40$, (f) une interaction significative entre la variable genre et la variable sexe, Lambda de Wilks $(6,1094)=.98, p<.05$, (g) une absence d'interaction significative entre les variables pratique sportive, sexe et genre, Lambda de Wilks $(6,1094)=.995, p=.85$.

Les tests univariés font apparaître : (a) un effet significatif au niveau de l'échelle de Masculinité : $F(1,562)=31.22, p<.0001$, les SPC $(M=4.98)$ obtenant un score plus élevé sur cette échelle que les SPNC ( $M=$ 4.47), et (b) un effet significatif au niveau de l'échelle de Féminité : $F(1,562)=4.92, p<$ .05 , les scores des SPC $(M=5.12)$ étant inférieurs à ceux des SPNC $(M=5.32)$.

\subsection{La pratique sportive en fonction du genre}

Les sujets pouvaient soit : (a) pratiquer des «Sports Masculins » $(\mathrm{SM})$, (b) pratiquer des « Sports Féminins » $(\mathrm{SF})$, (c) pratiquer des « Sports appropriés aux deux sexes » (SAMF), ou (d) ne pas pratiquer de sport [les « Non-Sport» (NS)]. Une première analyse de type Chi-carré fut appliquée aux données avec comme VI le genre des sujets, et comme VD le choix de la pratique sportive. Les résultats indiquent une répartition du choix des pratiques en fonction du genre des sujets qui s'éloigne trop de la distribution théorique pour être due au simple hasard de la distribution : $\chi^{2} \quad(9, \mathrm{~N}$ $=857)=164.82, p<.0001$.

L'analyse de la contribution des cases $a$ posteriori montre que les sujets Masculins sont sous-représentés dans les groupes SF, SAMF, et NS, et sur-représentés dans le groupe des pratiques SM. Les sujets Féminins sont sur-représentés dans les groupes SF et NS, et sous-représentés dans le groupe SM. Les sujets Androgynes sont sur-représentés dans les groupes SAMF et SF, tandis qu'ils sont sous-représentés dans le groupe NS. Les sujets Non-différenciés sont sous-représentés dans le groupe SM et sur-représentés dans le groupe NS (voir tableau V). La même différence dans la répartition suivant les groupes de pratiques sportives est mise en évidence pour le groupe des garçons : $\chi^{2}$ Garçons $(9, \mathrm{~N}=$ $402)=58.71, p<.0001$, et celui des filles : $\chi^{2}$ Filles $(9, \mathrm{~N}=455)=29.33, p<.001$. L'étude de la contribution des cases a posteriori révèle néanmoins des patrons de différenciation qui sont différents en fonction du sexe des sujets. Chez les garçons, les sujets Masculins sont sous-représentés dans le groupes SF, SAMF, et NS, et surreprésentés dans le groupe des pratiques SM. Les garçons Féminins sont sous-représentés dans le groupe SM. Les sujets Androgynes sont sur-représentés dans le groupe SF, tandis qu'ils sont sous-représentés dans le groupe des NS. Les garçons Non-différenciés sont sous-représentés dans les groupes SM et SF et sur-représentés dans le groupe NS et SAMF. Dans le groupe des filles, par contre, nous constatons que les sujets Masculins sont surreprésentés dans le groupe des pratiques SM. Les filles féminines sont sur-représentées dans le groupe NS, et sous-représentées dans le groupe SM. Les Androgynes sont sur-représentées dans le groupe SAMF, tandis qu'elles sont sous-représentées dans le groupe NS. On ne constate aucune différence de répartition (au seuil $p$ $<$.05) parmi les groupes de pratiques sportives chez les sujets Non-différenciés.

Il ne fut pas possible de mener une MANOVA avec le sexe, le genre et les pratiques sportives comme variables indépendantes car les effectifs dans certaines catégories étaient nuls (i.e., garçons ; nondifférenciés ; sports féminins). Néanmoins, afin d'explorer les liaisons entre le score des sujets aux sous-échelles Masculinité et Féminité et le choix des pratiques sportives, il fut conduit une MANOVA avec : (a) les pratiques sportives (NS, SM, SF, SAMF), (b) le sexe comme VI, et les scores des sujets aux échelles de Masculinité et de Féminité de la version courte pour adolescents français du BSRI comme VD. 
TABLEAU 5 : Répartition, selon le genre, des filles et des garçons dans les différents types d'activités sportives (en fréquence et en pourcentages).

\begin{tabular}{|c|c|c|c|c|c|}
\hline & & Masculin & Androgyne & Féminin & Non-différencié \\
\hline Garçons & Sports masculins (SM) & $102(58.96 \%)$ & $34(48.57 \%)$ & $9(25.00 \%)$ & $31(25.20 \%)$ \\
\hline \multirow[t]{4}{*}{$\mathrm{N}=402$} & Sports féminins (SF) & $4(2.31 \%)$ & $5(7.14 \%)$ & $1(2.78 \%)$ & $0(0.00 \%)$ \\
\hline & $\begin{array}{c}\text { Sports appropriés } \\
\text { aux deux sexes (SAMF) }\end{array}$ & $25(14.45 \%)$ & $21(30.00 \%)$ & $11(30.56 \%)$ & $41(33.33 \%)$ \\
\hline & Non-Sportif (NS) & $42(24.28 \%)$ & $10(14.29 \%)$ & $15(41.67 \%)$ & $51(41.46 \%)$ \\
\hline & Total & $173(100.00 \%)$ & $70(100.00 \%)$ & $36(100.00 \%)$ & $123(100.00 \%)$ \\
\hline Filles & Sports masculins(SM) & $7(18.42 \%)$ & $10(7.41 \%)$ & $5(2.81 \%)$ & $9(8.65 \%)$ \\
\hline \multirow[t]{4}{*}{$\mathrm{N}=455$} & Sports féminins (SF) & $9(23.68 \%)$ & $48(35.56 \%)$ & $55(30.90 \%)$ & $34(32.69 \%)$ \\
\hline & $\begin{array}{c}\text { Sports appropriés } \\
\text { aux deux sexes (SAMF) }\end{array}$ & $11(28.95 \%)$ & $40(29.63 \%)$ & $33(18.54 \%)$ & $18(17.31 \%)$ \\
\hline & Non-Sportif (NS) & $11(28.95 \%)$ & $37(27.41 \%)$ & $85(47.75 \%)$ & $43(41.35 \%)$ \\
\hline & Total & $38(100.00 \%)$ & $135(100.00 \%)$ & $178(100.00 \%)$ & $104(100.00 \%)$ \\
\hline Total & Sports masculins (SM) & $109(51.66 \%)$ & $44(21.46 \%)$ & $14(6.54 \%)$ & $40(17.62 \%)$ \\
\hline \multirow[t]{3}{*}{$\mathrm{N}=857$} & Sports féminins (SF) & $13(6.16 \%)$ & $53(25.85 \%)$ & $56(26.17 \%)$ & $34(14.98 \%)$ \\
\hline & $\begin{array}{c}\text { Sports appropriés } \\
\text { aux deux sexes (SAMF) }\end{array}$ & $36(17.06 \%)$ & $61(29.76 \%)$ & $44(20.56 \%)$ & $59(25.99 \%)$ \\
\hline & Non-Sportif (NS) & $53(25.12 \%)$ & $47(22.93 \%)$ & $100(46.73 \%)$ & $94(41.41 \%)$ \\
\hline
\end{tabular}

L'analyse multivariée révèle : (a) un effet principal de la variable modalité de pratique sportive significatif, Lambda de Wilks $(6,1696)=.96, p<.0001$, (b) un effet principal de la variable sexe significatif, Lambda de Wilks $(2,848)=.90, p<$ .0001 , (c) une interaction significative, Lambda de Wilks $(2,1696)=.97, p<.01$.

Pour la population totale, et pour la variable modalité de pratique, les tests univariés font apparaître : (a) un effet significatif au niveau de l'échelle de Masculinité : $F(3,853)=39.43, p<.0001$, les moyennes des groupes de sujets à cette échelle étant dans l'ordre décroissant : SM $(M=5.19)$, SAMF $(M=4.66)$, SF $(M=4.50)$, NS $(M=$ 4.17), et (b) un effet significatif au niveau de l'échelle de Féminité : $F(3,853)=$ $21.77, p<.0001$, les scores des sujets à cette échelle étant dans l'ordre décroissant : $\mathrm{SF}(M=5.62), \mathrm{NS}(M=5.26), \operatorname{SAMF}(M=$ $5.25), \mathrm{SM}(M=4.80)$. Des tests post-hoc de Newman-Keuls (au seuil $p<.05$ ) indiquent que sur l'échelle de Masculinité, les scores des sujets SF et SAMF ne sont pas significativement différents $(p=.15)$, tandis que tous les autres scores tous significativement différents quand on les compare deux à deux (i.e., $\mathrm{SM}>\mathrm{SAMF}=\mathrm{SF}>\mathrm{NS}$ ). Sur l'échelle de Féminité, les scores des sujets SAMF et NS ne se différencient pas ( $p=$ .87), les autres scores étant significativement différents de ceux des autres groupes quand on les compare deux à deux (i.e., SF $>$ SAMF $=$ NS > SM).

Deux MANOVAs complémentaires ont été réalisées en prenant séparément les filles et les garçons. Celles-ci font apparaître :

- Pour les garçons : Un effet multivarié significatif, Lambda de Wilks $(6,794)=.88$, $p<.0001$. Les analyses univariées révèlent : (a) un effet significatif au niveau l'échelle de Masculinité : $F(3,398)=$ $16.87, p<.0001$, les scores des sujets étant dans l'ordre décroissant : SAMF $(M=$ 
5.35), SM $(M=5.27), \mathrm{SF}(M=4.70), \mathrm{NS}$ $(\mathrm{M}=4.50)$, et (b) une absence d'effet significatif au niveau de l'échelle de Féminité : $F(3,398)=1.09, p=.35$. Des tests post hoc de Newman-Keuls (au seuil $p<.05$ ) menés sur les scores obtenus à l'échelle de Masculinité font apparaître que les scores des groupes SAMF et SM ne sont pas significativement différents. La même chose est observée pour les scores des groupes SF et NS. Les scores des groupes SM et SAMF sont significativement différents des scores des groupes SF et NS (i.e., $\mathrm{SAMF}=\mathrm{SM}>\mathrm{SF}=\mathrm{NS}$ ).

- Pour le groupe des filles : Un effet multivarié significatif, Lambda de Wilks $(6,900)=.89, p<.0001$. Les analyses univariées révèlent : (a) un effet significatif au niveau l'échelle de Masculinité : $F(3,451)$ $=12.19, p<.0001$, les scores des sujets étant dans l'ordre décroissant : SM $(M=$ 4.69), SAMF $(M=4.63), \mathrm{SF}(M=4.44)$, NS (3.95), et (b) un effet significatif au niveau de l'échelle de Féminité : $F(3,451)$ $=4.25, p<.01$, les scores des sujets à cette échelle étant dans l'ordre décroissant : NS $(M=5.68)$, SAMF $(M=5.67)$, SF $(M=$ 5.66), SM $(M=5.13)$. Pour l'échelle de Masculinité, des tests post hoc de Newman-Keuls font apparaître que seuls les scores du groupe NS sont différents (au seuil $p<.01)$ de ceux de tous les autres groupes, tandis que les scores des autres groupes ne différent pas significativement entre eux (au seuil $p>.05$ ) (i.e., $\mathrm{SM}=$ $\mathrm{SAMF}=\mathrm{SF}>\mathrm{NS}$ ). Sur l'échelle de Féminité, les scores du groupe SM sont différents (au seuil $p<.05$ ) de ceux de tous les autres groupes, alors qu'aucune différence significative n'est mise en évidence entre les scores des autres groupes (au seuil $p<$ .05) (i.e., $\mathrm{NS}=\mathrm{SAMF}=\mathrm{SF}>\mathrm{SM}$ ).

\subsection{Le champ des pratiques sportives selon le genre des sujets}

Afin d'étudier la distribution des pratiques sportives en fonction du genre, une Analyse en Composantes Multiples (ACM) fut réalisée à partir des variables sexe, genre, typage sexuel des pratiques sportives, et modalité de pratique (i.e., compétitive versus non-compétitive) (voir figure 1). L'ACM est une induction de l'Analyse Factorielle des Correspondances (AFC). Elle généralise le test d'indépendance du Chi-carré fait sur des tableaux de contingence (AFC; 2 variables qualitatives) en analysant des tableaux de plusieurs variables (Tableaux de Burt). En

FIGURE I : Mode d'organisation des pratiques sportives selon le genre et le sexe des sujets (Analyse en Composantes Multiples).

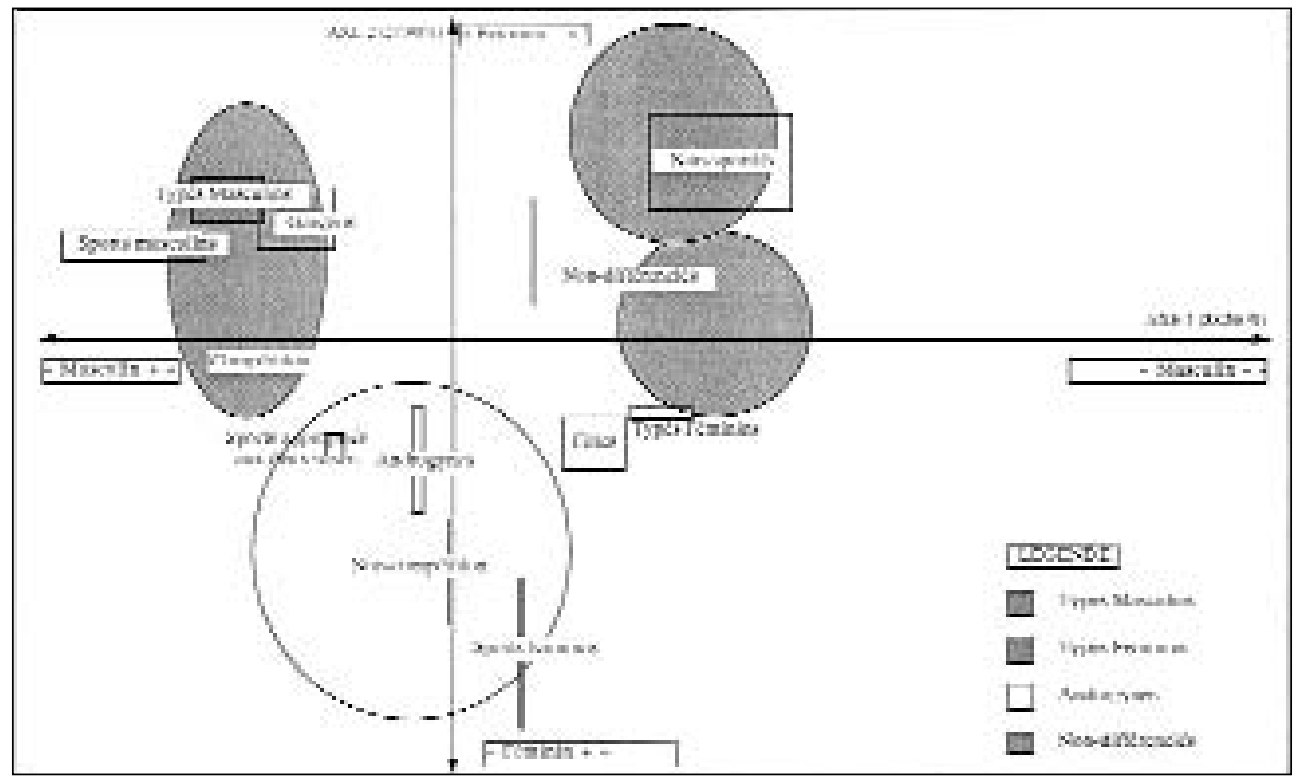

Note : Les coordonnées des individus et les contributions des modalités à chacun des axes sont disponibles auprès du premier auteur. 
d'autres termes, elle permet d'interpréter en même temps les relations entre plusieurs variables qualitatives, ou bien entre deux variables ayant de nombreuses modalités (Foucart, 1997).

Il n'a été procédé à aucune analyse typologique, puisque nos groupes étaient définis a priori par l'hypothèse formulée à partir de la répartition des sujets (en fonction de leur genre) sur les différentes modalités de pratiques sportives. Bien qu'il n'existe pas de règle déterminée pour choisir le nombre d'axes qui doivent être pris en compte pour la description des données, comme il est d'usage (Foucart, 1997), la variance importante expliquée par les deux axes $(67 \%)$ nous amène à penser que la représentation des deux premiers axes est suffisante dans le cadre de cette étude.

\section{DISCUSSION}

L'objet de cette étude était double : (a) confirmer que le sport est une activité masculine (cette différence marquant l'importance que ce domaine revêt aux yeux de certains sujets), (b) montrer que le genre des sujets organise aussi bien la fréquence, les modalités, mais également le type de pratique sportive. Si le sport est effectivement un objet perçu comme masculin, cette catégorisation sexuée doit s'actualiser différemment suivant les sujets. Plus généralement, nous nous interrogions sur l'effet du genre (ou de la sexuation psychologique) sur l'appropriation des activités physiques et sportives. Nous avions donc posé cinq hypothèses qui se trouvent globalement être confirmées.

La première question que nous pouvions nous poser était de savoir, si effectivement, et ce malgré la forte poussée féminine de ces trente dernières années, le sport était toujours une activité majoritairement pratiquée par les hommes, et donc pouvait encore être considéré comme un domaine masculin. Les résultats des analyses confirment cette conception. Les adolescents sont significativement plus nombreux que les adolescentes à faire du sport. Ils le sont encore plus quand il s'agit de comparer des modes de pratiques sportives (i.e., compétition $v s$ non-compétition). C'est ce que montre l'opposition marquée sur le premier axe de l'Analyse en Composantes Multiples (cf. figure 1.) entre le groupe « Fille-Non Compétition » et le groupe «GarçonCompétition ». Ces résultats confortent l'hypothèse de l'importance accordée à la pratique sportive dans la construction du Soi masculin dans nos sociétés occidentales (Cross \& Madson, 1997 ; Messner, 1988, 1990), et sont comparables aux travaux antérieurs qui qualifient le sport de « conservatoire d'une masculinité et fémininité des plus traditionnelles » (Louveau, in Davisse et Louveau, 1998, p.133).

L'analyse des données vient également confirmer l'influence du genre sur la valeur accordée à une activité (mesurée ici par le fait de pratiquer ou non). Sur la base des travaux de Bem (1981), nous avons émis l'hypothèse que les sujets possédant une forte identité de genre (i.e., Masculin, Féminin) valorisent les activités congruentes à leur identité, et évitent les activités noncongruentes avec celle-ci. Les résultats montrent que les sujets Masculins ou Féminins ont eu des comportements conformes aux stéréotypes sexués. Tout d'abord, les premiers pratiquent davantage le sport que les seconds, et ce, quel que soit leur sexe biologique. Ces tendances apparaissent accentuées quand on prend en compte la modalité de pratique (compétitive $v s$ non compétitive). En ce qui concerne l'influence du «typage sexuel » des activités physiques et sportives les résultats sont conformes à ce qui était attendu. Les sujets typés sexuellement (i.e., Masculin, Féminin) ont tendance à pratiquer des activités congruentes avec leur identité. Ainsi, quand il font du sport, les sujets Masculins sont plus nombreux que les sujets Féminins à pratiquer des activités sportives masculines, tandis que les sujets Féminins sont plus nombreux que les sujets Masculins à pratiquer des activités sportives féminines.

Nous nous attendions également à ce que les sujets qui n'emploient pas de manière systématique ou préférentielle les schèmes de catégorisation sexuelle (i.e., Androgynes, Non-différenciés) soient indifférents à la pratique d'activités sexuellement typées, ou davantage enclins à pratiquer des activités non-conformes à leur sexe (Matteo, 1986 ; Ostrow et al., 1981 ; Salminen, 1990). 
Les sujets Non-différenciés, se sont avérés moins sportifs que les Androgynes ou les Masculins. L'analyse du type de pratiques sportives (i.e., masculine, féminine, appropriée aux deux sexes) en fonction du genre des sujets confirme que les Androgynes et les Non-différenciés se répartissent globalement de manière plus équilibrée dans les différents groupes d'activités sportives, et pratiquent plus facilement que les sujets Masculins et/ou Féminins des sports qui ne sont pas nécessairement conformes à leurs stéréotypes de sexe.

Néanmoins, il ne faut pas envisager la catégorisation par genre de manière stricte, cela aboutissant à une «perte d'information » (e.g., Bem, 1977 ; les variables catégorielles apportant une moindre «quantité » d'information que les variables d'intervalles), mais plutôt comme un processus dynamique, susceptible d'évoluer. Bem suggère que les approches permettant d'identifier l'influence de chaque sous-échelle (i.e., Masculinité, Féminité) sur les variables étudiées devront être privilégiées. Dans de récents travaux portant sur la multi-dimensionnalité du concept de soi, des auteurs (Marsh \& Byrne, 1991; Spence \& Hall, 1996) soulignent également cette nécessité. Dans le cas de la pratique sportive, la plupart des auteurs avancent que ce sont les scores des sujets à la sous-échelle Masculine qui expliquent les différents patrons de comportements (Butcher, 1989 ; Colley, Roberts, \& Chipps, 1985 ; Gill, 1992). Nos résultats semblent appuyer ce modèle «masculin » de l'androgynie psychologique quand nous restons à un niveau global d'étude de la pratique sportive. Les sujets « Sportifs » ont des scores significativement supérieurs à ceux des « Non-sportifs » à la sous-échelle Masculine. Les résultats sont similaires concernant les sujets sportifs « Compétitifs » et « Non-compétitifs ». Les différences ne sont pas ou peu significatives concernant les scores à la sous-échelle Féminine entre ces différentes catégories de sujets. Cependant, les résultats sont différents dans les analyses conduites à partir du « typage sexuel » des activités physiques et sportives. Conformément à ce qu'avancent Marsh et Byrne (1991) dans leur approche « additive et différentielle » de l'androgynie psychologique, il semble que les scores des sujets aux deux sous-échelles du BSRI (M et
F) varient de manière substantielle en fonction de la sexuation de l'activité. Les sujets ayant le pointage le plus élevé à la souséchelle $\mathrm{M}$ sont ceux qui pratiquent des sports masculins ou appropriés aux deux sexes, tandis que ceux pour qui les scores à la sous-échelle F sont les plus hauts sont ceux qui pratiquent de activités sportives féminines. Cela est particulièrement vrai pour le groupe des Filles. Concrètement, cela signifie qu'on peut prédire le comportement des filles à partir de leur score sur la sous-échelle Féminine : plus le score est élevé, moins elles auront tendance à pratiquer une activité sportive ; et plus le score est bas, plus les sujets auront tendance à faire un sport masculin. Il serait intéressant d'examiner l'influence de cette même souséchelle sur les motifs de la pratique d'une activité sportive majoritairement pratiquée par les femmes (i.e., équitation, gymnastique, etc.). Des études seront sans doute nécessaires pour mettre en évidence les « processus » sous-jacents au choix des sujets concernant une activité sportive et ses modalités de pratique. Un modèle comme celui développé par Eccles et ses collaborateurs (1983), qui tient compte des attentes, mais aussi de la valeur de l'activité pour le sujet, pourrait servir de cadre à de futures recherches. Nous pourrions essayer de montrer, comme cela a déjà été fait concernant l'influence prépondérante du sexe sur le curriculum scolaire du sujet par le biais de différentes variables motivationnelles (Eccles, 1987 ; Duru-Bellat, 1994, 1995), que les mêmes mécanismes peuvent être évoqués pour le genre dans des cadres particuliers comme la pratique sportive de haut niveau, ou bien la pratique obligatoire de l'Éducation Physique et Sportive.

\section{BIBLIOGRAPHIE}

Alain, M. (1996). La mesure des rôles sexuels. Bulletin de Psychologie, 424, 396-404.

Archer, J., \& McDonald, M. (1990). Gender roles and sports in adolescent girls. Leisure Studies, 4, 225-241.

Arnaud, P., \& Terret, T. (1996). Histoire du sport féminin, tomes 1 et 2. Paris-Montréal : L'Harmattan.

Bem, S. L. (1977). On the utility of alternative procedures for assessing psychological 
androgyny. Journal of Consulting and Clinical Psychology, 45, 196-205.

Bem, S. L. (1981). Gender schema theory : A cognitive account of sex-typing. Psychological Review, 88, 354-364.

Bem, S. L. (1985). Androgyny and gender schema theory : A conceptual and empirical integration. In T. B. Sonderregger (Ed.), Nebraska Symposium on Motivation : Psychology and Gender, 32 (pp. 179-226). Lincoln University.

Bem, S. L., \& Lenney, E. (1976). Sex typing and avoidance a cross-sex behavior. Journal of Personality and Social Psychology, 33, 4854.

Blanchard-Fields, F., Suhrer-Roussel, L., \& Hertzog, C. (1994). A confirmatory factor analysis of the Bem Sex Role Inventory : Old questions, new answers. Sex Roles, 30, 423457.

Butcher, J. E. (1989). Adolescent girl's sex role development : Relationship with sport participation, self-esteem, and age at menarche. Sex Roles, 20, 575-593.

Carvalho, A.-M. A, Smith, P. K., Hunter, T., \& Costabile, A. (1990). Playground activities for boys and girls : Developmental and cultural trends in children's perceptions of gender differences. Play and Culture, 3, 343-347.

Clément, J.-P. (1981). La force, la souplesse et l'harmonie. Étude comparée de trois sports de combats (Lutte - Judo - Aïkido). In C. Pociello (Ed.), Sports et société. Approche socioculturelle des pratiques (pp. 285-301). Paris : Vigot.

Colley, A., Roberts, N., \& Chipps, A. (1985). Sex-role identity, personality and participation in team and individual sports by males and females. International Journal of Sport Psychology, 16, 103-112.

Colley, A., Nash, J., O’Donnel, L., \& Restorick, L. (1987). Attitudes of the female sex-role and sex-typing of physical activities. International Journal of Sport Psychology, 8, 19-29.

Constantinople, A. (1973). Masculinity-feminity : An exception to a famous dictum ? Psychological Bulletin, 80, 389-407.

Cronbach, L. (1951). Coefficient alpha and internal strucure of test. Psychometrika, 16, 296-334.

Cross, S. E., \& Madson, L. (1997). Models of the self : self-construals and gender. Psychological Bulletin, 122, 5-37.

Czisma, K. A., Wittig, A. F., \& Schurr, K. T. (1988). Sports stereotypes and gender. Journal of Sport and Exercise Psychology, 10, 62-74.
Davisse, A., \& Louveau, C. (1991). Sports, école, société. La part des femmes. Joinvillele-Pont : Actio.

Davisse, A., \& Louveau, C. (1998). Sports, école, société. La différence des sexes. Paris : L'Harmattan.

Deeter, T. E. (1989). Development of a model of achievement behavior for physical activity. Journal of Sport and Exercise Psychology, 11, 13-25.

Deeter, T. E. (1990). Re-modeling expectancy and value in physical activity. Journal of Sport and Exercise Psychology, 12, 86-91.

Duru-Bellat, M. (1994). Filles et garçons à l'école, approches sociologiques et psychosociales. $1^{\text {re }}$ Partie : Des scolarités sexuées, reflet des différences d'aptitudes, ou de différences d'attitudes? Revue Française de Pédagogie, 109, 111-141.

Duru-Bellat, M. (1995). Filles et garçons à l'école, approches sociologiques et psychosociales. $2^{\mathrm{e}}$ Partie : La construction scolaire des différences entre les sexes. Revue Française de Pédagogie, 110, 75-109.

Eccles, J. S. (1987). Gender roles and women's achievement-related decisions. Psychology of Women Quaterly, 11, 135-172.

Eccles, J. S., Adler, T. F., Futterman, R., Goff, S. B., Kaczala, C. M., Meece, J. L., \& Midgley, C. (1983). Expectancies, values, and academic behaviors. In J. T. Spence (Ed.), Achievement and achievement motivation (pp. 75146). San Francisco, CA : Freeman.

Eccles, J. S., Jacobs J. E., \& Harold R. D. (1990). Gender role stereotypes, expectancy effects, and parents' socialization of gender differences. Journal of Social Issues, 46, 183 201

Eccles, J. S., \& Harold, R. (1991). Gender differences in sport involvement: Applying the Eccles and al. model. Journal of Applied Sport Psychology, 3 (1), 7-35.

Fontayne, P., Sarrazin, Ph., \& Famose, J.-P. (2000). The Bem Sex-Role Inventory : Validation of a short-version for French teenagers. European Review of Applied Psychology, 50 (4), 405-416.

Foucart, T. (1997). L'analyse des données. Mode d'emploi. Rennes : PUR.

Gill, D. L. (1992). Gender and sport behavior. In T. Horn (Ed.), Advances in sport psychology (pp. 143-160). Champaign, IL : Human Kinetics.

Hall, E. G, Durborow, B., \& Progen, J. L. (1986). Self-esteem of female athletes and nonathletes relative to sex-role type and sport type. Sex Roles, 15, 379-390.

Harry, J. (1995). Sport ideology, attitudes toward women, and anti-homosexual attitudes. Sex Roles, 32, 109-116. 
Hurtig, M.-C., \& Pichevin, M.-F. (1986). La différence des sexes. Paris : Éditions Tierce.

Ignico, A. A. (1989). Development and verification of gender-role stereotyping index for physical activities. Perceptual and Motor Skills, 68, 1067-1075.

Kane, M. J. (1988). The female athletic role as a status determinant within the social systems of high school adolescents. Adolescence, $\mathbf{9 0}$, 253-264.

Kirckaldy, B., \& Furnham, A. (1990). Personality and sex differences in recreational choices. Sportwissenschaft, 20, 43-55.

Koivula, N. (1995). Rating of gender appropriateness of sports participation : Effects of gender based schematic processing. Sex Roles, 33, 543-547.

Marin, M. (1988). Gender-differences in sport and movement in Finland. International Review for Sociology of Sport, 23, 345-359.

Marsh, H. W., \& Byrne, B. M. (1991). Differenciated additive androgyny model : Relations between masculinity, feminity and multiple dimensions of the self-concept. Journal of Personality and Social Psychology, 61, 811-828.

Matteo, S. (1986). The effect of sex and genderschematic processing on sport participation. Sex Roles, 15, 417-432.

Matteo, S. (1988). The effect of gender-schematic processing on decisions about sex-inappropriate sport behavior. Sex Roles, 18, 41-58.

Meece, J. L., Parsons, J. L., Kaczala, C., Goff, S. B., \& Futterman, R. (1982). Sex differences in math achievement : Toward a model of academic choice. Psychological Bulletin, 91, 324348.

Messner, M. A. (1988). Sports and male domination: The female athlete as contested ideological terrain. Sociology of Sport Journal, 5, 197-211.

Messner, M. A. (1990). Men studying masculinity : Some epistemological issues in sport sociology. Sociology of Sport Journal, 7, 136153.
Metheny, E. (1965). Connotations of movement in sport and dance. W. C. Brown : Dubuque.

Ostrow, A. C., Jones, D. C., \& Spiker, D. D. (1981). Age role expectations and sex role expectations for selected sport activities. The Research Quaterly for Exercise and Sport, 52, 216-227.

Pociello, C. (1981). « La force, l'énergie, la grâce et les reflexes »; Le jeu complexe des dispositions culturelles et sportives. In C. Pociello (Ed.), Sports et société. Approche socioculturelle des pratiques (pp. 171-237). Paris : Vigot.

Pociello, C. (1995). Les cultures sportives. Paris : PUF.

Ryckman, R. M., \& Hamel, J. (1995). Male and female adolescents motive related to involvement in organized team sports. International Journal of Sport Psychology, 26, 383-397.

Salminen, S. (1990). Sex role and participation in traditionaly inappropriate sports. Perceptual and Motor Skills, 71, 1216-1218.

Spence, J. T., \& Hall, S. K. (1996). Children's gender related self-perceptions, activity preferences, and occupationnal stereotypes : A test of three models of gender constructs. Sex Roles, 35, 659-691.

Tap, P. (1985). Masculin et féminin chez l'enfant. Toulouse : Privat.

Vigarello, G. (1988). Une histoire culturelle du sport. Techniques d'hier et d'aujourd'hui. Paris : Revue EPS - Robert Laffont.

Wigfield, A., \& Eccles, J. S. (1992). The development of achievement task values : A theoretical analysis. Developmental review, 12, 265-310.

Wigfield, A., Harold, R. D., Freedman-Doan, C., Eccles, J. S., Yoon, K. S., Arbreton, A. J. A., \& Blumenfeld, P. C. (1997). Change in children's competence beliefs and subjective task values across the elementary school years : A 3-year study. Journal of Educational Psychology, 89, 451-469. 


\title{
Le pratiche sportive degli adolescenti : una differenziazione secondo il genere
}

Riassunto:

L'identità sessuale dei soggetti influenza il valore che questi ultimi assegnano alle attività (Eccles, Adler, Futterman, Goff, Kaczala, Meece \& Midgley, 1983). In questo studio proviamo a mostrare che la scelta delle pratiche sportive di 858 soggetti adolescenti (403 ragazzi e 455 ragazze), età media di 15.4 anni $(E T=0.83)$ è legato al loro profilo di genere $(B e m, 1981)$. Analisi statistiche (Chi2, MANOVAS, Analisi delle Componenti Multiple) furono realizzate a partire da un questionario nel quale i soggetti dovevano : a) rispondere ad una versione corta del BSRI (Fontane, Sarrazin \& Famose, in stampa), b) indicare il loro sesso, la loro pratica sportiva e la modalità di questa pratica (competitiva / non competitiva). I risultati mostrano che la percezione di uno sport come conforme ai ruoli sessuati maschile o femminile influenza la scelta delle pratiche. I soggetti « mascolini »e « femminini » sceglieranno degli sport congruenti e rifiuteranno gli sport non-congruenti con la loro identità, mentre i soggetti « androgini » $\mathrm{e}$ « non-differenziati » rivelano più facilmente delle scelte non-conformi agli stereotipi culturali del loro sesso.

Parole chiave : genere, sport, valore.

\section{Las prácticas deportivas en los adolescentes : diferencia según el género}

\begin{abstract}
Resumen :
La identidad sexual de los sujetos influencia el valor que aquellos conceden a las actividades (Eccles, Adler, Futterman, Goff, Kaczala, Meece, \& Midgley, 1983). En este estudio, nosotros tratamos de mostrar que la elección de prácticas deportivas de 858 sujetos adolescentes (403 niños y 455 niñas), con edad media de 15,4 años ( $E T=0,83$ ) esta ligada a su perfil de género (Bem, 1981). Análisis estadísticos (Chi2, MANOVAS, Análisis en componentes multiples) fueron realizados a partir de un cuestionario en el que los sujetos debian (a) responder a una version corta del BSRI (Fontayne, Sarrazin, \& Famose, bajo prensa), (b) indicar su sexo, su practica deportiva y la modalidad de esta practica (competitiva/ no-competitiva). Los resultados muestran que la percepción de un deporte como conformado para los roles sexuales masculino o femenino, influencia la elección de practicas. Los sujetos « masculinos » $\mathrm{y}$ 《 femininos » elijen deportes congruentes y rechazan el deporte no-congruente con su identidad, mientras que los sujetos androginos y no-diferenciados, revelan de manera más comoda elecciones no-conformes a los esteorotipos culturales de su sexo.
\end{abstract}

Palabras claves : deporte, género, valor.

\section{Sportpraxis von Jugendlichen : Eine Differenzierung nach dem Geschlecht}

\section{Zusammenfassung :}

Die Geschlechtsidentität der Versuchspersonen beeinflusst den Wert, den sie diesen Aktivitäten zuordnen (Eccles, Adler, Futtermann, Goff, Kaczala, Meece\& Midgley, 1983). In dieser Studie wollen wir zeigen, dass die Wahl einer sportlichen Aktivität von 858 Jugendlichen (403 Jungen und 455 Mädchen) mit einem Durchschnittsalter von 15,4 Jahren $(\mathrm{s}= \pm 0,83)$ von ihrem Geschlechtsprofil abhängt (Bem, 1981). Ein Fragebogen, in dem die Versuchspersonen a) eine Kurzversion des BSRI (Fontayne, Sarrazin \& Famose im Druck) beantworten und b) Angaben zu ihrem Geschlecht, ihrer sportlichen Aktivität und der Art ihrer sportlichen Aktivität (wettkampfmäßig, nicht wettkampfmäßig) angeben mussten, wurde statistischen Analysen (Chi2, MANOVAs, Faktorenanalyse) unterzogen. Die Resultate zeigen, dass die Einschätzung eines Sports hinsichtlich seiner Konformität mit den männlichen oder weiblichen Geschlechterrollen, die Wahl der Praktiken beeinflusst. Die « männlichen » und «weiblichen » Versuchspersonen wählen entsprechende Sportarten und weisen Sportarten zurück, die nicht zu ihrer Geschlechtsidentität passen. " Androgyne » oder « undifferenzierte » Versuchspersonen, treffen eher Wahlen, die nicht mit den kulturellen Stereotypen ihres Geschlechts übereinstimmen. 